\title{
Transforming growth factor- $\beta 1$ in the microenvironment of ischemia reperfusion-injured kidney enhances the chemotaxis of mesenchymal stem cells to stromal cell-derived factor-1 through upregulation of surface chemokine (C-X-C motif) receptor 4
}

\author{
XIAO-YUN SI, JING-JING LI, TAO YAO and XIAO-YAN WU \\ Department of Nephrology, Zhongnan Hospital of Wuhan University, Wuhan, Hubei 430071, P.R. China
}

Received August 8, 2013; Accepted February 12, 2014

DOI: $10.3892 / \mathrm{mmr} .2014 .1989$

\begin{abstract}
Acute renal failure is one of the most common complications observed in hospitals. Although extensive studies have been carried out to search for therapeutic treatments, no effective cure has been established. In recent years, stem cell therapy for tissue engineering and repair has become a key area of study. Bone marrow mesenchymal stem cells (MSCs) have been demonstrated to exhibit a reparative role in ischemia reperfusion-injured kidney tissue, and the stromal cell-derived factor-1 (SDF-1)/chemokine (C-X-C motif) receptor 4 (CXCR4) axis has been found to play an important role in the migration and homing of MSCs. In the present study, transforming growth factor- $\beta 1$ (TGF- $\beta 1$ ) in the homogenate of the acute ischemia reperfusion-injured renal tissue was found to markedly increase the CXCR4 surface expression of MSCs, which contributes to the migration of MSCs to SDF-1. Neutralization of TGF- $\beta 1$ inhibited the migration in an antibody concentration-dependent manner, through downregulation of CXCR4 localized to the membrane. These observations suggest a potential mechanism for MSC migration to the kidney which may provide a possible therapeutic target for curing acute renal failure.
\end{abstract}

\section{Introduction}

Acute renal ischemia reperfusion injury is the main cause of acute kidney failure, resulting in a delay in kidney transplants and can result in transplant misfunction (1). Effective treatment remains hard to find, but the homing of mesenchymal stem cells (MSCs) to the kidneys has been shown to confer a

Correspondence to: Dr Xiao-Yun Si, Department of Nephrology, Zhongnan Hospital of Wuhan University, No. 163 Donghu Road, Wuchang, Wuhan, Hubei 430071, P.R. China

E-mail: sixiaoyun303@163.com

Key words: acute renal failure, mesenchymal stem cells, renal ischemia reperfusion, transforming growth factor- $\beta 1$, chemokine (C-X-C motif) receptor 4, stromal cell-derived factor-1 protective effect from renal ischemia reperfusion injury (2). MSCs have a strong replication capacity and multipotential differentiation ability. The metastasis and homing of MSCs are strongly associated with the axis of stromal cell-derived factor-1 (SDF-1)/chemokine (C-X-C motif) receptor 4 (CXCR4) (3). SDF-1 is a CXC chemokine, also termed CXC ligand 12. At present, CXCR4 is the only known receptor for SDF-1. The binding of SDF-1 to CXCR4 is the basis for a number of biological effects (4-6).

In previous years, CXCR4 expression has been observed in MSCs, which may facilitate the homing activity of MSCs to the bone marrow along an SDF-1 gradient (7). The in vitro migration ability of MSCs is enhanced as the concentration of SDF-1 increases, and the CXCR4 inhibitor, AMD3100, is able to disturb the migration ability of the MSCs. Using an acute renal ischemia failure model, Tögel et al found that SDF-1 expression was markedly upregulated in injured tissue of an ischemia group, compared with a normal rat group (8). There was simultaneous migration of CXCR4-positive bone marrow stem cells to regions of renal ischemia. This suggests a potential role for bone marrow stem cell transplants in the treatment of acute renal injury due to ischemia reperfusion.

The regulation of SDF-1 and/or CXCR4 is thought to control the migration and targeting of MSCs. Although MSCs in the bone marrow constitutively express high levels of CXCR4, only a small portion of cells present CXCR4 at the membrane and only a small amount (2-17\%) of total CXCR4 is located on the membrane (9). Thus, increasing the surface presentation of CXCR4 is likely to be important for regulation of MSC homing activity, and thus may cure injured tissues. Transforming growth factor- $\beta 1$ (TGF- $\beta 1$ ) is a cytokine upregulated during kidney ischemia and hypothesized to be a marker of recovery for post-ischemic kidney tissue (10). TGF- $\beta 1$ can modulate the SDF-1/CXCR4 axis and therefore controls the migration of various cell types (11-16). MSCs are similar to tumor cells and cells of mesenchymal origin, with regard to biological characteristics and specific molecular mechanisms. The present study tested the hypothesis that TGF- $\beta 1$ can mediate the SDF-1/CXCR4 axis, thereby inducing MSC homing activity. These results may shed light on the recovery treatment for acute renal ischemia reperfusion injury. 


\section{Materials and methods}

Experimental animals and antibodies. Female specific pathogen-free level Sprague Dawley (SD) rats (weight, $180 \mathrm{~g}$ ) were obtained from the Experimental Animal Centre of Wuhan University (Wuhan, China). All animal experimental protocols were approved by the Animal Care and Use Committee of Wuhan University. Fusin (V340) antibody, specific to the C-X-C family, was a product of Bioworld Technology, Inc. (St. Louis Park, MN, USA). Monoclonal TGF- $\beta 1$ antibody was purchased from R\&D Systems (Minneapolis, MN, USA). Antibodies for MSC characterization were from Biolegend, Inc. (San Diego, CA, USA).

Isolation and culture of bone marrow MSCs. Bilateral thigh bones were taken under aseptic conditions from 2-3-week-old normal female SD rats. Bone marrow was flushed out by serum-free low glucose Dulbecco's modified Eagle's medium (DMEM) after two washes with phosphate-buffered saline (PBS). Next, Percoll lymphocyte separating medium was added (at a 1:1 ratio) and cells were isolated according to the combined method of adherent cell isolation and density gradient centrifugation. Mononuclear cells were absorbed from the cell interface for inoculation. Cells were cultured at $37^{\circ} \mathrm{C}$ in a humidified incubator with $5 \% \mathrm{CO}_{2}$. After $48-72 \mathrm{~h}$, the nonadherent cells were removed. Following this, culture medium was renewed every other day and cells with a confluency of $\sim 80 \%$ were subcultured.

Characterization of isolated bone marrow MSCs and CXCR4-positive rate detection. The second passage MSCs were used for flow cytometry (FCM) analysis. The MSC surface markers, CD29, CD105, CD34 and CD45, were analyzed. Antibodies for CD34 and CD45 were labeled with fluorescein isothiocyanate, while antibodies for CD29 and CD105 were labeled with phycoerythrin (PE). Cell surface CXCR4 was detected by PE-labeled antibody through FCM.

Preparation of tissue homogenate of ischemia reperfusion-injured renal tissue. Kidney ischemia reperfusion was performed under standard conditions. The two renal pedicles were clamped for $40 \mathrm{~min}$ and reperfusion was subsequently performed for $60 \mathrm{~min}$. Following this, kidney homogenate was prepared in PBS buffer at a final concentration of $20 \mathrm{~g} / \mathrm{l}$. The homogenate was centrifuged at a speed of $500 \mathrm{x} \mathrm{g}$ for $15 \mathrm{~min}$. Supernatant was collected and filtered using a $0.22-\mu \mathrm{m}$ filter. Aliquots were stored at $-70^{\circ} \mathrm{C}$ for further use. SD rats of $\sim 8$ weeks old were used for experiments carried out under aseptic conditions.

Quantitative polymerase chain reaction ( $q P C R)$ and western blotting (WB) detection of CXCR4 protein levels. MSC total RNA was extracted by TRIzol reagent (Invitrogen Life Technologies, Carlsbad, CA, USA). cDNA was synthesized using the RevertAid H Minus First Strand cDNA Synthesis kit (Fermentas, Waltham, MA, USA) and $2 \mu \mathrm{g}$ RNA templates were applied. Using SYBR Green PCR master mix (Invitrogen Life Technologies), qPCR was performed and products were detected on a Step One Plus Real-Time PCR system (Invitrogen Life Technologies). A $100 \mathrm{nM}$ primer concentration was selected for the PCR system and $\beta$-actin was used as the internal reference. Primers used were as follows: CXCR4 forward, 5'-GCTAACACTTACGCAAAGACAT-3' and reverse, 5'-CGTGAAACAGACAAACAACAG-3'; $\beta$-actin forward, 5'-CGTTGACATCCGTAAAGACCTC-3' and reverse, 5'-TAGGAGCCAGGGCAGTAATCT-3'. PCR was performed for 40 cycles of $15 \mathrm{sec}$ at $95^{\circ} \mathrm{C}, 15 \mathrm{sec}$ at $59^{\circ} \mathrm{C}$ and $45 \mathrm{sec}$ at $72^{\circ} \mathrm{C}$. CXCR4 protein levels were determined by WB following normal protocols. Cells were lysed with buffer containing 50 $\mathrm{mM}$ Tris $\mathrm{HCl}$ (pH 7.5), $5 \mathrm{mM}$ EDTA, $150 \mathrm{mM} \mathrm{NaCl}, 0.5 \%$ Triton X-100, $10 \mathrm{mM}$ sodium fluoride, $20 \mathrm{mM}$ 2-ME, $250 \mu \mathrm{M}$ sodium orthovanadate, $1 \mathrm{mM}$ phenylmethylsulfonyl fluoride and complete protease inhibitor mixture (Sigma-Aldrich, St. Louis, MO, USA), and were incubated at $4^{\circ} \mathrm{C}$ for $1 \mathrm{~h}$. The lysates were ultrasonicated and centrifuged at $12,000 \mathrm{x} \mathrm{g}$ for $10 \mathrm{~min}$. Protein concentrations were determined using the BCA method. Proteins $(50 \mu \mathrm{g})$ were separated on eight $10 \%$ polyacrylamide sodium dodecyl sulfate gels and electroblotted onto nitrocellulose membranes (Hybond ECL; Amersham Pharmacia Biotech, Piscataway, NJ, USA). Subsequent to blocking with Tris-buffered saline and 5\% nonfat dried milk for $2 \mathrm{~h}$, the membrane was incubated overnight at $4^{\circ} \mathrm{C}$ with antibodies specific to the CXC family (Bioworld Technology, Inc., St. Louis Park, MN, USA) followed by incubation with a horseradish peroxidase-conjugated secondary antibody (goat anti mouse; 1:2,000; Pierce, Rockford, IL, USA) for $45 \mathrm{~min}$ at room temperature, and the signals were visualized by enhanced chemiluminescence detection. As a loading control, the blots were reprobed with a specific antibody against human $\beta$-actin (mouse anti human; 1:5,000; Santa Cruz Biotechnology, Inc., Santa Cruz, CA, USA).

MSC migration assay. MSC migration was evaluated using a 24-well chamber system. In total, $20 \mu \mathrm{g}$ Matrigel was added to the top chamber prior to injection of MSCs $\left(3 \times 10^{4} / \mathrm{ml}\right)$. Next, $600 \mu 1$ DMEM containing $10 \%$ fetal calf serum with SDF-1 $\alpha$ $(100 \mathrm{ng} / \mathrm{ml})$ was applied in the bottom chamber. Various contents were added to the top chamber and cells were incubated at $37^{\circ} \mathrm{C}$ for $12 \mathrm{~h}$. Next, the Matrigel and cells above the microporous film were removed. Cells which had migrated to the lower surface were fixed by pure ethanol for $20 \mathrm{~min}$, stained by DAPI and photographed and counted under fluorescence microscopy.

Statistical analysis. Statistical significance was assessed by a two-tailed Student's t-test or analysis of variance test. $\mathrm{P}<0.05$ was considered to indicate a statistically significant difference and $\mathrm{P}<0.01$ was considered to indicate a marked statistically significant difference.

\section{Results}

Morphology of isolated MSCs. Freshly inoculated MSCs presented a round shape of non-uniform size in the suspension. After $72 \mathrm{~h}$, the number of adherent monolayer fibroblasts with a fusiform shape increased. On day 14, cells reached $90 \%$ confluence. Following this, cells were passaged in a 1:3 ratio. After $24 \mathrm{~h}$, passage cells had adhered and exhibited a rapid rate of proliferation. The majority of cells presented a tenuous spindle shape, and protuberant pancake and fibroblast-like shapes were also observed. The third passage of cells with a 

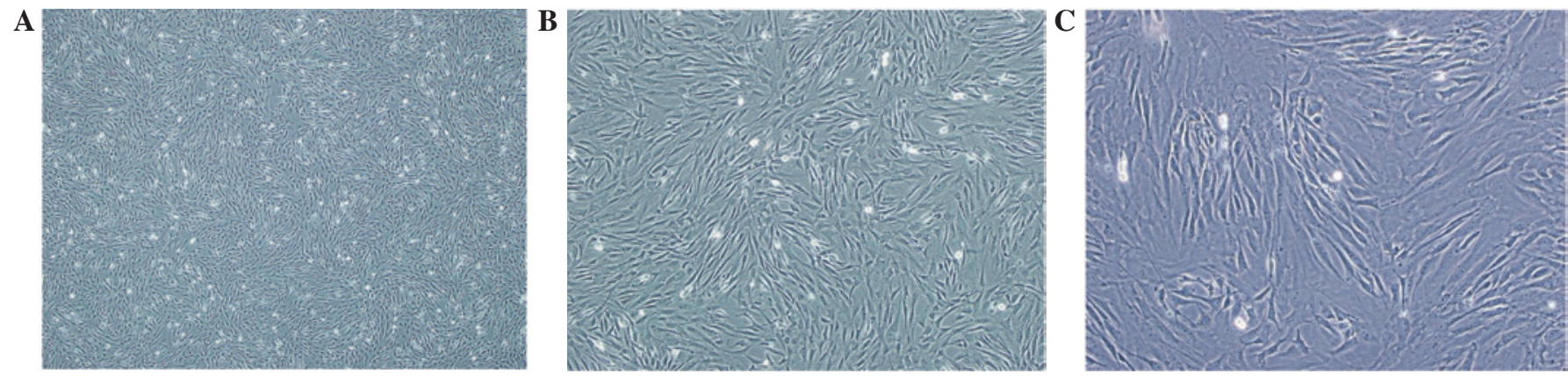

Figure 1. Morphology of isolated bone marrow mesenchymal stem cells. (A) Cells of passage 3 on day 2 (magnification, x40). (B) Cells of passage 4 on day 1 (magnification, x100). (C) Cells of passage 5 on day 3 (magnification, $\mathrm{x} 200$ ).
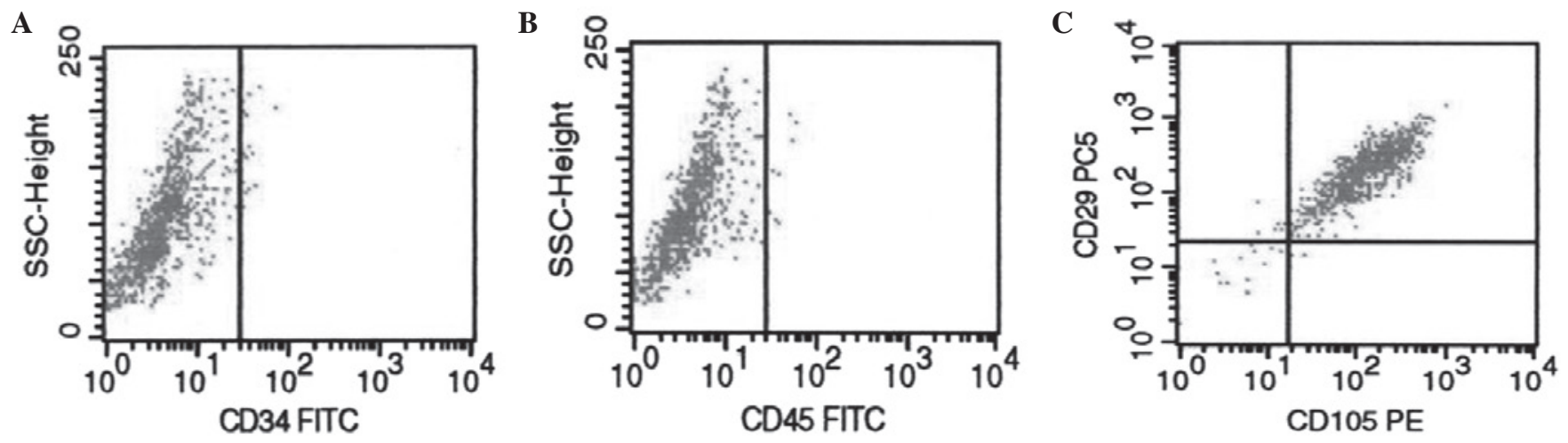

Figure 2. Mesenchymal stem cell characterization of isolated cells. Cells from passage 2 were used for flow cytometry analysis. (A) CD34, (B) CD45 and (C) CD29 and CD105 were analyzed by flow cytometry. SSC, side scatter detector; FITC, fluorescein; PE, phycoerythrin.
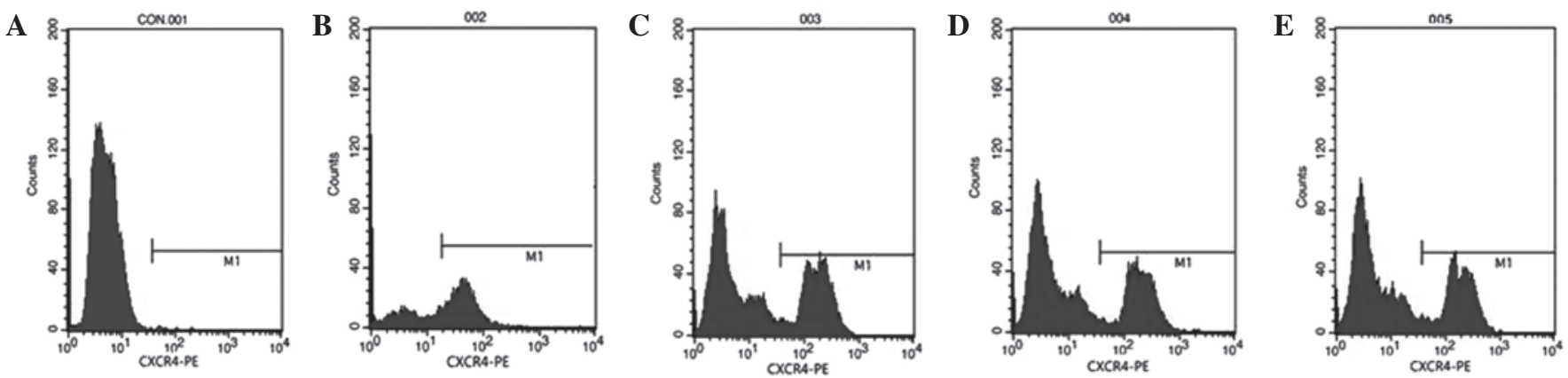

Figure 3. MSC surface CXCR4-positive rate determination. (A) Negative control (primary antibody was replaced by phosphate-buffered saline), (B) Normal MSCs (MSC group), (C) MSCs incubated with homogenate (H group), (D) MSCs incubated with homogenate and CXCR4 antibody (H\&Cab group) and (E) MSCs incubated with homogenate and tumor growth factor- $\beta 1$ antibody (H\&Tab group). Cells were subsequently incubated with CXCR4-specific antibody and analyzed by flow cytometry. CXCR4, chemokine (C-X-C motif) receptor 4; PE, phycoerythrin; MSC, mesenchymal stem cell.

tenuous spindle shape demonstrated good refractivity. The cell boundary was clear and nuclei were plump in the center. Cells were arranged with a relative directivity which exhibited a radial or vortex pattern. Cells in subsequent passages were similar to those of passage 3, with a uniform size and a tenuous spindle shape (Fig. 1).

Characterization of MSCs. Cells of passage 2 were used for analysis and MSC surface markers, CD29, CD105, CD34 and CD45, were applied for MSC characterization. Labeled antibodies were incubated with cells and continued FCM analysis was performed. The results demonstrated a $97.18 \%$ positive rate for CD29 and CD105, while the positive rate for CD34 and CD45 was 1.37 and $0.95 \%$, respectively. These results are characteristic of MSC surface features (Fig. 2).

TGF- $\beta 1$ enhances the MSC CXCR4-positive expression rate. MSCs were subjected to various treatments and FCM analysis was carried out (Fig. 3). The results showed that normal MSCs presented CXCR4 on the membrane (MSC group relative fluorescence intensity, 5.89). The negative control group demonstrated low fluorescence (the primary antibody was replaced by $\mathrm{PBS}$ ). Homogenate from the ischemia reperfusion renal tissue markedly increased the CXCR4 surface 
Table I. Number of migrated MSCs in the groups.

\begin{tabular}{lc}
\hline Group & Migrated MSCs, cells $/ \mathrm{cm}^{2}$ \\
\hline MSCs & $18.47 \pm 5.02$ \\
$\mathrm{H}$ & $38.92 \pm 6.79$ \\
$\mathrm{H} \& \mathrm{Tab}(1 \mu \mathrm{g} / \mathrm{ml})$ & $24.28 \pm 4.37$ \\
$\mathrm{H} \& \mathrm{Tab}(5 \mu \mathrm{g} / \mathrm{ml})$ & $16.11 \pm 3.68$ \\
H\&Tab $(10 \mu \mathrm{g} / \mathrm{ml})$ & $13.26 \pm 4.03$ \\
H\&Cab & $7.03 \pm 2.14$ \\
\hline
\end{tabular}

Data are presented as the mean \pm standard deviation $(n=3)$. MSC, mesenchymal stem cell; H, MSCs incubated with homogenate; H\&Tab, MSCs incubated with homogenate and tumor growth factor- $\beta 1$ antibody; H\&Cab, MSCs incubated with homogenate and CXCR4 antibody.

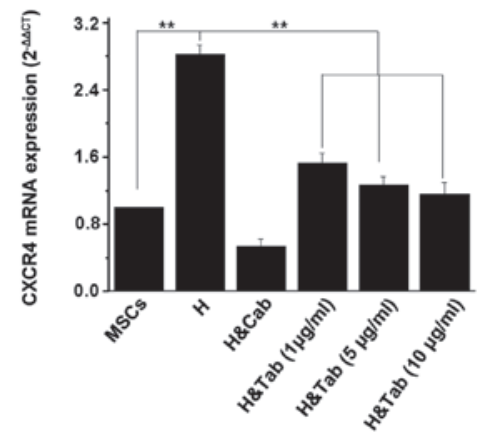

Figure 4. qPCR results of CXCR4 mRNA level changes. MSCs were treated with various combinations of components and total mRNA was extracted for qPCR. mRNA expression is presented as the amplification multiple $\left(2^{-\Delta \Delta C t}\right)$ ${ }^{* *} \mathrm{P}<0.01$. MSCs, mesenchymal stem cells; $\mathrm{H}$, MSCs incubated with homogenate; H\&Cab, MSCs incubated with homogenate and CXCR4 antibody; H\&Tab, MSCs incubated with homogenate and tumor growth factor- $\beta 1$ antibody CXCR4, chemokine (C-X-C motif) receptor 4; qPCR, quantitative polymerase chain reaction.

A

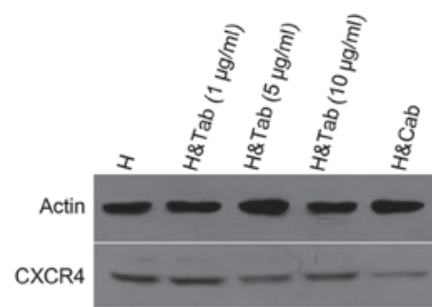

B

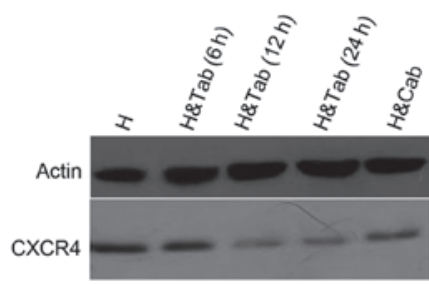

Figure 5. Analysis of CXCR4 protein level variations by WB. MSCs were treated with various combinations of components and $\mathrm{WB}$ was carried out to detect CXCR4 protein levels. (A) TGF- $\beta 1$ antibody concentration gradients were applied. (B) The time course of the effect of TGF- $\beta 1$ is shown. H, MSCs incubated with homogenate; H\&Cab, MSCs incubated with homogenate and CXCR4 antibody; H\&Tab, MSCs incubated with homogenate and tumor growth factor- $\beta 1$ antibody; CXCR4, chemokine (C-X-C motif) receptor 4 ; WB, western blotting; TGF- $\beta 1$, transforming growth factor- $\beta 1$; MSC, mesenchymal stem cells.
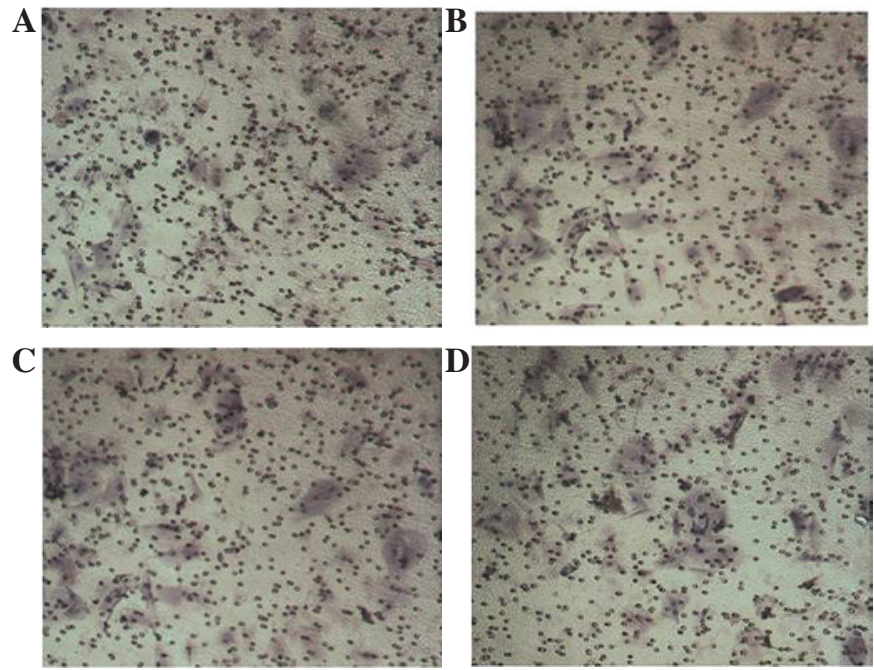

$\mathbf{E}$
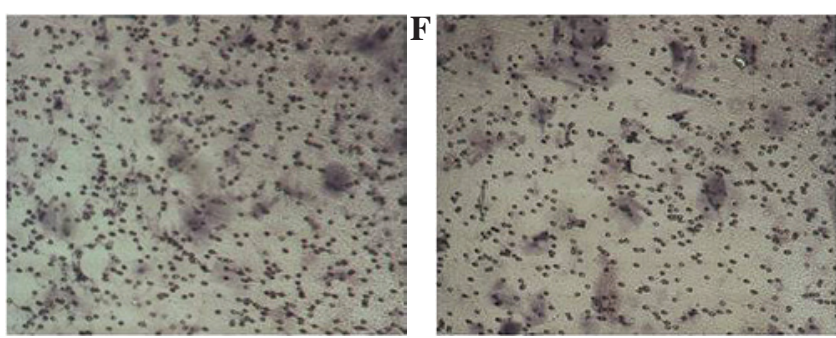

Figure 6. Evaluation of MSC migration by a 24-well chamber system. (A-F) Representative images of the groups MSCs, H, H\&Tab $(1 \mu \mathrm{g} / \mathrm{ml})$, $\mathrm{H} \& \mathrm{Tab}(5 \mu \mathrm{g} / \mathrm{ml}), \mathrm{H} \& \mathrm{Tab}(10 \mu \mathrm{g} / \mathrm{ml})$ and $\mathrm{H} \& \mathrm{Cab}$, respectively. A detailed description of each group is presented in Table I. MSC, mesenchymal stem cell; H, MSCs incubated with homogenate; H\&Tab, MSCs incubated with homogenate and tumor growth factor- $\beta 1$ antibody; $\mathrm{H} \& \mathrm{Cab}$, MSCs incubated with homogenate and CXCR4 antibody.

presentation (homogenate group relative fluorescence intensity, 6.22). This increased presentation was inhibited by TGF- $\beta 1$ antibody (TGF- $\beta 1$ antibody group relative fluorescence intensity, 4.89), which indicates that TGF- $\beta 1$ in the homogenate can promote CXCR4 surface presentation. In addition, the positive expression rate may be greatly reduced by CXCR4 antibody (CXCR4 group relative fluorescence intensity, 0.88).

CXCR4 expression is promoted by TGF- $\beta 1$ in the homogenate. qPCR results revealed that homogenate markedly upregulated CXCR4 mRNA levels $(\mathrm{P}<0.01)$ compared with the MSC group. Cells treated with homogenate and CXCR4 antibody demonstrated a small difference from the MSC group (no statistical significance). TGF- $\beta 1$ antibody partially neutralizes TGF- $\beta 1$ in the homogenate, which can decrease CXCR4 mRNA levels. The degree of inhibition depends on the antibody concentration (Fig. 4). WB of CXCR4 protein levels in various groups was performed following treatment. Homogenate markedly increased the CXCR4 protein levels, while TGF- $\beta 1$ neutralization downregulated protein levels, as with mRNA. Inhibition was antibody concentration- (Fig. 5A) and time-dependent (Fig. 5B). CXCR4 antibody can decrease CXCR4 protein levels, which corresponds in part to mRNA levels following antibody treatment.

TGF- $\beta 1$ enhances the migration of MSCs. Normal MSCs also exhibitmigration ability to SDF-1 $\left(18.47 \pm 5.02 \mathrm{cells} / \mathrm{cm}^{2}\right)$ (Fig.6). 
Homogenate from ischemia reperfusion renal tissue markedly increased MSC migration to SDF-1 $\left(38.92 \pm 6.79\right.$ cells $\left./ \mathrm{cm}^{2}\right)$. TGF- $\beta 1$ antibody decreased the effects caused by homogenate, in a concentration-dependent manner (Table I). CXCR4 antibody markedly inhibited MSC migration $\left(7.03 \pm 2.14\right.$ cells $\left./ \mathrm{cm}^{2}\right)$, which suggests that the interaction of CXCR4 and SDF-1 plays an important role in renal ischemia reperfusion-induced MSC chemotaxis.

\section{Discussion}

Acute renal failure is a common clinical complication in hospitals. In patients requiring dialysis, acute renal failure is associated with a high rate of in-hospital mortality $(17,18)$. Among the causes of acute renal failure, acute ischemia reperfusion is the most common cause of kidney failure. Much effort has been paid to the development of therapies for the disease but an effective cure is yet to be developed. In previous years, stem cells have become a key area of study for tissue repair and engineering, due to their multipotential differentiation and infinite proliferation ability (19). Human bone marrow MSCs have already been found to accelerate the recovery of acute renal injury in mice (20), however, the mechanism for the migration of MSCs to the kidneys remains unclear. The regulation of MSC migration is thus likely to be crucial for the repair of injured kidneys.

The SDF-1/CXCR4 axis has been shown to function in the migration control of a number of cell types. SDF-1 is responsible for the in vitro migration of melanoma cells, and migration may also be promoted by TGF- $\beta 1$. This is due to the upregulation of CXCR4 by TGF- $\beta 1$, which interacts with SDF-1, which in turn contributes to cell migration (21). During acute renal ischemia reperfusion injury, upregulation of various cytokines has been observed. These factors are mostly likely responsible for tissue repair. In the present study, homogenate of the ischemia reperfusion injured renal tissue was found to enhance the migration of bone marrow MSCs induced by SDF-1. The migration of MSCs to SDF-1 may be inhibited when TGF- $\beta 1$ in the homogenate is neutralized by specific antibodies. Inhibition was in an antibody concentration- and time-dependent manner. FCM analysis of cell surface CXCR4 indicates that TGF- $\beta 1$ may increase surface CXCR4 expression, which leads to increased cell migration to SDF-1. In addition, the total CXCR4 mRNA and protein levels were increased by the homogenate or TGF- $\beta 1$. These results indicate that TGF- $\beta 1$ may enhance the migration of MSCs induced by SDF-1, through upregulation of MSC surface CXCR4 expression. This suggests a possible ischemia reperfusion-injured kidney repair mechanism of MSC induction, induced by tissue-secreted SDF-1. This may aid the development of therapy for acute ischemia reperfusion renal injury, which is currently associated with high mortality in hospitals.

\section{Acknowledgements}

This study was supported by a grant from the Natural Science Foundation of Hubei Province Science and Technology Program (no. 2009CDB428), China.

\section{References}

1. Weight SC, Bell PRF and Nicholson ML: Renal ischaemia - reperfusion injury. Br J Surg 83: 162-170, 2005.

2. Herrera MB, Bussolati B, Bruno S, Fonsato V, Romanazzi GM and Camussi G: Mesenchymal stem cells contribute to the renal repair of acute tubular epithelial injury. Int J Mol Med 14: 1035-1041, 2004

3. Peled A, Petit I, Kollet O, et al: Dependence of human stem cell engraftment and repopulation of NOD/SCID mice on CXCR4. Science 283: 845-848, 1999.

4. Ratajczak MZ,Zuba-Surma E, Kucia M, Reca R, Wojakowski W and Ratajczak J: The pleiotropic effects of the SDF-1-CXCR4 axis in organogenesis, regeneration and tumorigenesis. Leukemia 20: 1915-1924, 2006.

5. Teicher BA and Fricker SP: CXCL12 (SDF-1)/CXCR4 pathway in cancer. Clin Cancer Res 16: 2927-2931, 2010.

6. Petit I, Jin D and Rafii S: The SDF-1-CXCR4 signaling pathway: a molecular hub modulating neo-angiogenesis. Trends Immunol 28: 299-307, 2007.

7. Wynn RF, Hart CA, Corradi-Perini C, et al: A small proportion of mesenchymal stem cells strongly expresses functionally active CXCR4 receptor capable of promoting migration to bone marrow. Blood 104: 2643-2645, 2004.

8. Tögel F, Isaac J, Hu Z, Weiss K and Westenfelder C: Renal SDF-1 signals mobilization and homing of CXCR4-positive cells to the kidney after ischemic injury. Kidney Int 67: 1772-1784, 2005.

9. Uchida D, Onoue T, Kuribayashi N, Tomizuka Y, Tamatani T, Nagai $\mathrm{H}$ and Miyamoto Y: Blockade of CXCR4 in oral squamous cell carcinoma inhibits lymph node metastases. Eur J Cancer 47: 452-459, 2011.

10. Docherty NG, Pérez-Barriocanal F, Balboa NE and López-Novoa JM: Transforming growth factor-betal (TGF-beta1): a potential recovery signal in the post-ischemic kidney. Ren Fail 24: 391-406, 2002.

11. Ao M, Franco OE, Park D, Raman D, Williams K and Hayward SW: Cross-talk between paracrine-acting cytokine and chemokine pathways promotes malignancy in benign human prostatic epithelium. Cancer Res 67: 4244-4253, 2007.

12. Kojima Y, Acar A, Eaton EN, et al: Autocrine TGF-beta and stromal cell-derived factor-1 (SDF-1) signaling drives the evolution of tumor-promoting mammary stromal myofibroblasts. Proc Natl Acad Sci USA 107: 20009-20014, 2010.

13. Zhao H and Peehl DM: Tumor-promoting phenotype of CD90hi prostate cancer-associated fibroblasts. Prostate 69: 991-1000, 2009.

14. Zhao XP, Huang YY, Huang Y, et al: Transforming growth factor-beta1 upregulates the expression of CXC chemokine receptor 4 (CXCR4) in human breast cancer MCF-7 cells. Acta Pharmacol Sin 31: 347-354, 2010.

15. Katoh M and Katoh M: Integrative genomic analyses of CXCR4: transcriptional regulation of CXCR4 based on TGFbeta, Nodal, Activin signaling and POU5F1, FOXA2, FOXC2, FOXH1, SOX17, and GFI1 transcription factors. Int J Oncol 36: 415-420, 2010.

16. Bertran E, Caja L, Navarro E, et al: Role of CXCR4/SDF-1 alpha in the migratory phenotype of hepatoma cells that have undergone epithelial-mesenchymal transition in response to the transforming growth factor-beta. Cell Signal 21: 1595-1606, 2009.

17. Lameire N, Van Biesen W and Vanholder R: Acute renal failure. Lancet 365: 417-430, 2005.

18. Waikar SS, Curhan GC, Wald R, et al: Declining mortality in patients with acute renal failure, 1988 to 2002. J Am Soc Nephrol 17: 1143-1150, 2006.

19. Kørbling M and Estrov Z: Adult stem cells for tissue repair - a new therapeutic concept? N Engl J Med 349: 570-582, 2003.

20. Morigi M, Introna M, Imberti B, et al: Human bone marrow mesenchymal stem cells accelerate recovery of acute renal injury and prolong survival in mice. Stem Cells 26: 2075-2082, 2008.

21. Bartolomé RA, Gálvez BG, Longo N, et al: Stromal cell-derived factor-1alpha promotes melanoma cell invasion across basement membranes involving stimulation of membrane-type 1 matrix metalloproteinase and Rho GTPase activities. Cancer Res 64: 2534-2543, 2004. 